\title{
Transatlantica
}

Revue d'études américaines. American Studies Journal

Amérique militante

Didier Combeau, Des Américains et des armes à feux. Violence et démocratie aux Etats-Unis. Saul Cornell, A Well-Regulated Militia: The Founding Fathers and the Origins of Gun Control in America.

Paris : Belin, 2007. New York : Oxford University Press, 2006.

\section{Renaud Pacoud}

\section{OpenEdition}

Journals

Édition électronique

URL : http://journals.openedition.org/transatlantica/4033

DOI : $10.4000 /$ transatlantica.4033

ISSN : $1765-2766$

Éditeur

AFEA

Référence électronique

Renaud Pacoud, «Didier Combeau, Des Américains et des armes à feux. Violence et démocratie aux EtatsUnis. Saul Cornell, A Well-Regulated Militia: The Founding Fathers and the Origins of Gun Control in America. », Transatlantica [En ligne], 1 | 2008, mis en ligne le 28 juillet 2008, consulté le 29 avril 2021. URL : http://journals.openedition.org/transatlantica/4033; DOI : https://doi.org/10.4000/ transatlantica. 4033

Ce document a été généré automatiquement le 29 avril 2021.

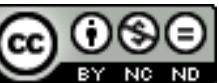

Transatlantica - Revue d'études américaines est mis à disposition selon les termes de la licence Creative Commons Attribution - Pas d'Utilisation Commerciale - Pas de Modification 4.0 International. 


\title{
Didier Combeau, Des Américains et des armes à feux. Violence et démocratie aux Etats-Unis. Saul Cornell, A Well-Regulated Militia: The Founding Fathers and the Origins of Gun Control in America.
}

Paris : Belin, 2007. New York : Oxford University Press, 2006.

\author{
Renaud Pacoud
}

1 Deux ouvrages différents dans leur approche mais également passionnants permettent de saisir l'évolution du débat sur les armes à feu aux États-Unis. Des Américains et des armes à feux. Violence et démocratie aux Etats Unis de Didier Combeau rend compte de la relation complexe que les Américains entretiennent avec les armes à feu depuis la fondation de la nation et de la polémique contemporaine sur la question du droit aux armes. A Well-Regulated Militia: The Founding Fathers and the Origins of Gun Control in America de Saul Cornell propose une fascinante histoire intellectuelle et légale du Deuxième amendement qui renouvelle sensiblement notre regard sur cette mystérieuse disposition du Bill of Rights et renvoie dos à dos les partisans des interprétations collective et individuelle.

2 L'ouvrage de Didier Combeau est sans doute le plus accessible pour le public français, auquel il est par ailleurs naturellement destiné. Il offre un panorama très clair de tous les aspects du débat sur les armes sans prendre partie et en posant comme objectif de recherche la restitution de la controverse plus que l'étude du fait social qui la suscite. Ce parti pris est en soi une garantie de neutralité qui n'est pas un luxe au vu des clichés parfois navrants qui circulent de ce côté-ci de l'Atlantique à propos des Américains et de leurs armes. 
3 L'auteur voit dans la persistance d'un accès particulièrement facile aux armes la résultante du fonctionnement particulier de la démocratie aux États-Unis et de ce qu'il implique au plan du rapport entre les citoyens, l'État et la collectivité. Dans ce jeu de relations complexe mais globalement cohérent et fonctionnel, le droit aux armes s'apparente tout autant à un droit des anciens qu'à une liberté des modernes et fait donc figure de garantie civique fondamentale. Didier Combeau décèle ici un trait distinctif de « l'identité américaine » qui fait du droit aux armes un élément constitutif de la démocratie.

4 La première partie du livre offre une efficace histoire des armes aux États-Unis en soulignant que le lien entre droit aux armes et américanité se fait dès les origines de la nation. L'auteur suggère par ailleurs que les controverses sur la meilleure interprétation du Deuxième amendement ne rendent pas vraiment compte de la nature $\mathrm{du}$ problème, puisque le droit individuel à s'armer est en réalité très largement coextensif du droit collectif aux armes garanti aux États, selon la lecture traditionnelle de l'amendement. Cette clarification, même si elle a le mérite de souligner que c'est l'anachronisme éventuel d'une telle garantie dans un État moderne qui pose problème davantage que la détermination objective de la nature du droit en question, passe néanmoins sous silence la trajectoire singulière du Deuxième amendement dans la pensée constitutionnelle américaine et les raisons historiques ayant conduit à la réactualisation constante de ce faux débat. Mais là n'était évidemment pas le projet. L'auteur insiste davantage sur l'héritage mythique de l'Ouest américain et son prolongement dans le droit à l'autodéfense accordé par de nombreux États pour montrer en quoi cette liberté laissée aux citoyens doit se comprendre comme une garantie de leur participation active au maintien de l'ordre.

Néanmoins, Didier Combeau montre avec force que cette conception toute particulière $\mathrm{du}$ civisme se paie chèrement. Il nous rappelle que «ce droit à combattre la violence par la violence [...] se traduit par un nombre d'homicides justifiables élevé sur le plan national » (43), et que les armes à feux sont incontestablement « l'outil de choix » dans la cadre de la participation des citoyens à la lutte contre la délinquance et la criminalité. Ce simple constat suggère que l'on est bien en présence ici « d'une autre peine de mort, à usage privé et expéditif » (43).

6 Du reste cette absence de monopolisation de la violence légitime - ou plus exactement, selon la distinction subtile établie par l'auteur, " de sa mise en oeuvre » puisque «le privilège de dire la loi, et donc de définir les conditions d'usage de la force demeure bien monopolisé par les pouvoirs publics » (175) - agit comme un puissant facteur d'hostilité instinctive aux projets de réglementation de la vente et de l'utilisation des armes. Depuis la loi Sullivan passée en 1911 par l'État de New York jusqu'aux initiatives du président Bill Clinton, force est de constater que l'arsenal législatif et réglementaire aux niveaux fédéré et fédéral reste qualitativement bien en deçà de ce qu'une société qui souhaiterait véritablement marginaliser les armes mettrait en place à cet effet. Les facteurs institutionnels lourds tels que l'absence de pouvoirs de police au niveau fédéral et la segmentation des approches législatives selon que l'on souhaite réglementer l'accès à certaines armes, les modes d'acquisition ou plus rarement les modalités d'utilisation ont également agi structurellement contre les ambitions des partisans du contrôle. Selon l'auteur, « les lois adoptées aux États-Unis ne sont pas moins nombreuses qu'ailleurs mais se distinguent par leurs lacunes. Ils ne s'agit pas d'effets pervers qui apparaissent à l'usage mais bien de vices constitutifs qui sont le 
résultat de l'âpreté de la négociation collective : la plupart des textes sont des mesures de compromis qui permettent aux amateurs d'armes comme aux partisans de leur contrôle de crier victoire d'une façon ou d'un autre » (80).

7 Cette situation est à mettre en rapport avec la polémique qui s'est développée entre partisans du contrôle et défenseurs du droit individuel à s'armer depuis les années 1970. Dans la deuxième partie du livre, Didier Combeau montre de manière convaincante que les batailles de chiffres à laquelle se livrent les deux camps n'ont pas réellement contribué à éclairer le public sur la dangerosité ou l'utilité des armes à feu $\mathrm{Au} v \mathrm{vu}$ des conditions de production et de réception de ces données statistiques, on ne peut que remarquer que ces dernières ne favorisent pas la prise de décisions politiques un tant soit peu consensuelles et que la profusion de chiffres agit comme un écran puissant sur la structuration idéologique du débat. Il y aurait en l'occurrence deux visions distinctes de la société ne pouvant pas vraiment cohabiter : l'une postulant la centralité de la responsabilité individuelle et l'autre l'impératif de santé publique. La première fait des armes le symbole et le moyen de la liberté et de l'égalité des individus, et la seconde pose que la violence est une pathologie du corps social qui doit être diagnostiquée et traitée en tant que telle.

8 À cela s'ajoute la manière dont le système politique américain facilite la mise sous pression des élus au bénéfice de lobbys bien organisés et la difficulté pour l'État fédéral de faire valoir un intérêt supra étatique. Le système de représentation au Congrès des États-Unis et l'élection au suffrage indirect avec winner takes all du président augmentent en effet le pouvoir des États ruraux les moins peuplés, plus prompts à s'opposer à toute tentative de réglementation nationale. A la protection "symbolique " du Deuxième amendement s'ajoute donc celle, autrement plus efficace et puissamment endogène, de la structure du système politique américain. Les solutions nationales au problème des armes à feu seront difficiles à trouver.

9 La dernière partie du livre est sans doute la plus stimulante. Didier Combeau y prolonge sa réflexion sur l'articulation entre démocratie, américanité et droit aux armes à feu en faisant pièce à la thèse selon laquelle la culture des armes à feu serait le fruit d'un « attachement sentimental et contingent» (164) résultant de singularités historiques et démographiques américaines. La persistance d'une culture des armes est plutôt le fruit d'une « défiance vis-à-vis de l'institutionnalisation de la résolution des conflits » (171) fondée sur l'idée que les forces de police ne sont pas en mesure à elles seules de garantir la sécurité des citoyens. Cette conviction, qui unit les Afro-Américains des quartiers défavorisés et les militants de la National Rifle Association, valide l'argument utilitaire en faveur des armes et permet de faire légitimement le lien entre autodéfense et bien commun, entre liberté individuelle et nécessité publique. Le problème reste pourtant que la libre circulation des armes augmente considérablement «la difficulté que les autorités policières éprouvent à maintenir l'usage de la violence légale à un niveau acceptable par l'opinion publique » (184) et qu'elle rend la délinquance plus meurtrière et par conséquent plus insupportable.

Didier Combeau ne manque pas de souligner que, dans un tel contexte, le mode d'organisation de la société qui découle de l'idéologie des détenteurs d'armes conduit inévitablement à une "externalisation de l'enjeu, qui consiste à rejeter hors des frontières de la communauté tous ceux qui sont susceptibles de contribuer à la transformation d'un droit individuel en problème social »(201). Les mesures prises pour limiter l'accès aux armes des populations supposées dangereuses - c'est-à-dire 
celles qui sont les plus exposées à un environnement criminogène - s'apparentent à un processus d'exclusion particulièrement impitoyable. En effet, les barrières mises à l'exercice d'un droit constitutionnel à s'armer conçu comme fondamental et d'un droit commun à l'autodéfense considéré comme tout à fait élémentaire conduisent à un déni de citoyenneté évident. En reconnaissant le droit aux armes à feux comme constitutif de la citoyenneté politique tout en en limitant l'accès à certaines catégories de la population la société américaine fait des groupes sociaux défavorisés les débiteurs de la « créance » politique qui apparaît lorsque le droit de porter une arme se heurte au droit d'être protégé non seulement de la criminalité induite par leur libre circulation, mais aussi des interventions indues des pouvoirs publics (un problème récurrent lorsque la police est concurrencée dans ses prérogatives par les particuliers).

11 Il y aurait encore beaucoup à dire tant cette étude est riche et éclairante. On aurait pu attendre, néanmoins, un rappel historique plus conséquent sur la trajectoire du droit aux armes au XIXe siècle. On regrettera peut être aussi que l'auteur ait passé sous silence dans son chapitre sur la polémique entre partisans du droit aux armes et ceux de leur contrôle le retour depuis une vingtaine d'année, à l'initiative de la National Rifle Association, d'une controverse historique et juridique très vive autour du sens originel du Deuxième amendement. Un rappel des faits sur ce point aurait eu l'avantage de montrer que, pour partiellement factice que soit cette polémique, elle a surtout servi à populariser les thèses les plus radicales sur l'existence d'un droit constitutionnel indiscutable de posséder des armes à titre privé. De ce point de vue, l'activation d'un discours très revendicatif axé sur la reconquête d'une garantie inscrite dans le Bill of Rights suggère que le sens du Deuxième amendement n'est probablement pas moins politiquement contingent que celui d'autres garanties constitutionnelles, passées au rang de libertés suprêmes suite aux efforts de groupes de pression pour en étendre la portée. Didier Combeau affirme donc peut-être un peu vite qu'il «frappe du sceau constitutionnel l'union entre droit aux armes et identité nationale » (21). Cela est sans doute vrai dans l'esprit des détenteurs d'armes, mais il ne faut pas perdre de vue que cette charge symbolique, si puissante soit-elle, n'est pas naturelle et immuable, même pour une garantie de la Déclaration des droits. Le retour du Deuxième amendement au rôle de la Cour suprême des États-Unis presque 70 ans après la dernière intervention des juges sur la question, dans l'arrêt United States v. Miller (1939) est là pour le rappeler. La Cour a en effet récemment accédé à la requête du District of Columbia d'examiner en appel une décision d'une cour fédérale inférieure invalidant intégralement le District of Columbia Firearms Control Regulations Act de 1975. Cette loi est connue aux Etats-Unis pour être la plus rigoureuse en matière de contrôle des armes à feux puisqu'elle interdit à quelques exceptions près la détention et la vente d'armes dites "de poing " (handguns) et contraint les détenteurs de fusils et de carabines à décharger et démonter leurs armes (ou éventuellement à les cadenasser) lorsqu'ils ne les utilisent pas. En acceptant l'appel la Cour Suprême a formulé la question de droit qu'elle souhaitait résoudre en des termes qui semblent déjà postuler l'existence d'un droit individuel à s'armer: "Whether the [provisions of the DC code] violate the Second Amendment rights of individuals who are not affiliated with any state-regulated militia, but who wish to keep handguns and other firearms for private use in their homes? » Il est donc peu probable que United States v. Miller, qui avait validé le National Firearms Act de 1934 au bénéfice d'une lecture collective - à l'époque très consensuelle - du Deuxième amendement pèse bien lourd sur la conscience de la nouvelle majorité conservatrice à la Cour. Le contenu du débat constitutionnel a profondément changé à l'instigation des 
détenteurs d'armes, et des juges conservateurs à l'activisme pas toujours bien tempéré ne sauraient l'ignorer.

La lecture de l'ouvrage de l'historien américain Saul Cornell, A Well-Regulated Militia : the Founding Fathers and the Origins of Gun Control in America ne peut justement que conforter le lecteur français dans son incrédulité face à l'aspect caricatural de ce débat sur le sens du Deuxième amendement. Si l'on suit cet historien - et la démonstration est convaincante - en dépit du nombre considérable de page écrites sur le sujet, ni les partisans d'une lecture individualiste de l'amendement ni leurs détracteurs collectivistes n'en offrent une interprétation fidèle à son sens originel, dont pourtant ils se réclament les uns comme les autres.

Saul Cornell voit dans la notion de "liberté bien administrée » (well-regulated liberty) le cadre dans lequel les pères fondateurs concevaient le droit à s'armer. Il s'agissait de garantir la sécurité des institutions et d'assurer la pérennité de la liberté politique nouvellement conquise sans avoir à instituer une armée permanente (standing army). Il en découle selon lui que le Deuxième amendement protège un droit « civique », conçu avant tout comme une obligation militaire envers la communauté. Les détenteurs de ce droit sont des citoyens au même titre qu'ils le sont lorsque les autorités publiques convoquent un jury lors d'un procès.

Le lecteur béotien peut estimer que cette représentation du Deuxième amendement ne diffère pas nettement de celle offerte par l'école du droit collectif. Saul Cornell souligne toutefois que les citoyens ne sont pas l'équivalent d'individus réunis collectivement en milice. La conception civique du droit de porter des armes interdit en effet de penser qu'il s'applique aux situations de création spontanée d'entités paramilitaires non prévues par la loi. Il s'ensuit que l'exercice de ce droit ne saurait s'envisager sans un contrôle strict effectué par les États dans le cadre de leurs pouvoirs de police, mais garantit également que l'autorité publique ne s'arrogera jamais le droit de désarmer ses administrés. La régulation par les pouvoirs publics n'est pas la condition contre laquelle s'échangerait la reconnaissance d'un droit inaliénable, elle lui est consubstantielle.

Mais c'est lorsque l'effort d'historicisation reprend le dessus sur le débat strictement originaliste-qui concerne avant tout la recherche des intentions originelles des rédacteurs de la Constitution et du Bill of Rights - que l'ouvrage devient le plus intéressant. Dans le chapitre sur l'époque jacksonienne l'auteur suggère que c'est dans bien dans le développement d'une culture individualiste dans ce contexte de révolution commerciale qui précède la guerre civile qu'il faut chercher l'origine de la confusion contemporaine entre droit aux armes et droit à l'autodéfense. L'intérêt pour la régulation et le contrôle des armes par les États qui se fait jour au même moment suscite aussi les premières défenses explicites du droit individuel à s'armer au motif de l'autodéfense : « Americans began sporting weapons designed primarily for self-defense [...] While many citizens outfitted themselves with these weapons, others recoiled at their countrymen penchants for traveling armed and demanded that their legislatures take strong measures to regulate, and in some cases to prohibit, this practice. The enactment of those first gun control measures prompted a backlash that produced the first systematic defense of an individual right to bear arms in self-defense » (137-138). En outre, Saul Cornell montre en quoi la lecture civique du Deuxième amendement a pesé après le passage du Quatorzième amendement, lorsque l'établissement de "milices noires" (negro militias) apparut aux républicains comme un rempart aux désordres civils qui régnaient dans le sud. Dans diverses procédures faisant suite à des 
tentatives de désarmement de ces milices par des membres du Ku Klux Klan la Cour suprême des États-Unis devait finalement consacrer la lecture antifédéraliste du Deuxième amendement proposée par les démocrates, à rebours des théories de l'incorporation ${ }^{1}$ défendues par certains républicains, qui voyaient dans le Quatorzième amendement la possibilité de faire appliquer aux États les garanties du Bill of Rights et donc de faire du droit constitutionnel de porter des armes un des ces "privileges and immunities » de la citoyenneté nationale protégés par la clause d'égale protection des lois. Dès lors, la théorie du droit collectif des États ne fit que gagner du terrain jusqu'à la décision historique United States v. Cruikshank de 1875: "The Court asserted in unambiguous terms that bearing arms for lawful purposes was not identical to the right protected by the Second Amendment that linked bearing arms to the participation in a militia » (194-195). En d'autres termes, la Cour reconnaissait que le droit commun de s'armer et celui protégé par le Deuxième amendement étaient juridiquement distincts et que l'objet de ce dernier n'était que de prévenir tout désarmement des milices d'État par le pouvoir fédéral. Ce faisant, elle substituait une lecture très étroite du Deuxième amendement à la conception civique originelle, comme elle devait le faire, de manière moins affirmée, dans l'arrêt United States v. Miller de 1939.

Revenant à la fin du livre sur le débat contemporain, Saul Cornell reconnaît que la dichotomie droit collectif/droit individuel, du fait de son simplisme historique, est un frein à une évolution plus constructive du débat, tout en admettant que la conception civique originelle souffre d'un anachronisme fatal qui la rend largement inopérante pour éclairer la controverse actuelle sur le contrôle approprié des armes à feu. L'auteur se risque néanmoins à préciser quelles régulations seraient envisageables si l'on suivait les prescriptions de sa lecture civique de l'amendement: «Registration, safe storage laws and limited ban on certain types of weapons with no connection to the goal of supporting a well-regulated militia are all consistent with this original vision; wholesale gun prohibition or domestic disarmament is not » (216). Tout resterait donc affaire d'équilibrage entre liberté de s'armer et impératif de sécurité publique. Mais, dans un contexte où le droit très consensuel à l'autodéfense armée fait peser un lourd soupçon d'illégitimité sur toute mesure de contrôle un tant soit peu restrictive, ce mieux disant régulateur paraît au pire hypocrite, au mieux particulièrement illusoire et inconscient des logiques d'exclusion qu'il recèle et des présupposés idéologiques dont celles-ci procèdent. Saul Cornell, partisan déclaré du contrôle des armes, ne semble pas du tout saisir l'impensé culturel de son propos lorsqu'il affirme quelques lignes plus loin : « Formulating an effective regulatory scheme that can promote public safety and that recognizes the radically different roles guns play in different American subcultures will not be easy. We currently have a national market in which guns sold in Virginia easily find their way to the Bronx. The gun cultures of Virginia and the Bronx are radically different however, and restrictions that might be prudent in one region might seem intrusive in the other " (216). Osera t-on lui conseiller de lire l'ouvrage de Didier Combeau? 


\section{NOTES}

1. La théorie de l'incorporation pose que les garanties du Bill of Rights s'appliquent aux États à travers la clause de procédure légale régulière et la clause d'égale protection du Quatorzième amendement. Cette théorie a été en grande partie adoptée par la Cour suprême des États-Unis.

INDEX

Thèmes : Recensions

\section{AUTEUR}

RENAUD PACOUD

ATER, Institut d'Études Politiques de Strasbourg 